\title{
Curcumin and Inflammatory Bowel Disease: Biological Mechanisms and Clinical Implication
}

\author{
Tauseef Ali $^{\mathrm{a}-\mathrm{c}}$ Faiz Shakir $^{\mathrm{a}} \quad$ Jordan Morton ${ }^{\mathrm{b}}$ \\ a Section of Digestive Diseases and Nutrition, at ${ }^{b}$ Department of Internal Medicine, University of Oklahoma Health \\ Sciences Center, and ' VA Medical Center, Oklahoma City, Okla., USA
}

\section{Key Words}

Curcumin - Turmeric - Inflammatory bowel disease $\cdot$ Herbal therapy

\begin{abstract}
Increased recognition of the limits of conventional medicine has helped drive the growing interest in complementary and alternative medicine which is now being commonly used in patients with chronic diseases, including individuals with Crohn's disease and ulcerative colitis. Recently, scientific interest has unraveled the beneficial pharmacological effects of curcumin. We present an updated concise review of currently available in vitro, animal and clinical studies demonstrating the therapeutic effect of herbal medication in inflammatory bowel disease.

Copyright $\odot 2012$ S. Karger AG, Basel
\end{abstract}

\section{Introduction}

Inflammatory bowel disease (IBD), which most commonly includes Crohn's disease (CD) and ulcerative colitis (UC), is believed to be due to the dysregulation of host immune responses leading to chronic intestinal inflammation in genetically susceptible populations. Therapeutic options for the management of IBD include amino- salicylates, antibiotics, corticosteroids, antimetabolite immunomodulators (e.g., 6-mercaptopurine, azathioprine and methotrexate), and biologic therapies such as anti-tumor necrosis factor (anit-TNF) agents (e.g., infliximab, adalimumab, certolizumab pegol) and natalizumab [1-3]. However, clinical efficacy is limited because of the lower rate of sustained remission and the increased risks of serious infections and malignancies with certain agents such as anti-TNF agents [4-6]. Increased recognition of the limits of conventional medicine has helped drive the growing interest in complementary and alternative medicine (CAM) which is now being commonly used by the general public and patients with chronic diseases [7], including individuals with CD and UC [8].

The use of curcumin, a yellow pigment widely used as a coloring agent and spice in many foods, predates ancient times and remains a vital ingredient in Ayurvedic and Chinese medicine. Recently, scientific interest has unraveled its beneficial pharmacological effects; they include antioxidant, anti-inflammatory, anticarcinogenic [9-11], hypocholesterolemic [12], antibacterial [13], wound-healing, antispasmodic, anticoagulant, antitumor [14] and hepatoprotective [15] activities. However, clinical applications in certain inflammatory diseases including IBD remain largely limited to case studies and small clinical trials.

\section{KARGER}

Fax +4161306 1234

E-Mail karger@karger.ch

www.karger.com
(C) 2012 S. Karger AG, Basel

0012-2823/12/0854-0249\$38.00/0

Accessible online at:

www.karger.com/dig
Tauseef Ali, MD, Assist. Prof. of Medicine

Section of Digestive Diseases and Nutrition

University of Oklahoma Health Sciences Center

Oklahoma City, OK 73104 (USA)

Tel. +1 405271 5428, E-Mail tauseef-ali@ouhsc.edu 


\section{Methods}

Literature searches were conducted in PubMed, Ovid, EMBASE and Cochrane Library databases in accordance with published recommendations [16-18]. Databases were used to search English language literature using the search terms curcumin, turmeric, inflammatory bowel disease, Crohn's disease, and ulcerative colitis. Both human and animal studies were reviewed and data from these studies were either included or interpreted in the current review.

\section{Curcumin and Immune Function}

Many studies have suggested beneficial effects of curcumin in vitro and in rodent models of chemically induced colitis [15, 19-31] (table 1). Curcumin significantly improves survival and colonic morphology, dampens local cytokine and chemokine production and reduces mucosal neutrophil infiltration. Curcumin modulates inflammation by downregulating genes involved in oxidative stress and fibrogenesis pathways. In the first study using genome-wide expression, mdrla $^{-/-}$mice fed a diet containing curcumin had lower colonic histologic injury scores than those fed a control diet [26]. The authors suggested that the favorable effect of curcumin on colonic inflammation likely resulted from upregulation of xenobiotic metabolism and a downregulation of proinflammatory pathways.

The anti-inflammatory properties of curcumin is attributed to its interference with the arachidonic acid cascade [32] and blocking nuclear factor (NF)- $\mathrm{\kappa B}$ activity which is implicated in the regulation of proinflammatory enzymes, including cyclooxygenase 2, 5-lipoxygenase and inducible nitric oxide synthase. The inhibition of NF- $\kappa \mathrm{B}$ is of particular importance and is considered a putative target for intervention in IBD [12,33], since the induction of NF- $\kappa \mathrm{B}$ by various signaling pathways is pivotal in the pathogenesis of IBD. One pathway through which NF- $\mathrm{KB}$ is induced in IBD is through the activation of Toll-like receptor 4 isoform [34]. Levels of Toll-like receptor 4 and NF- $\mathrm{\kappa B}$ proteins in inflamed tissue are suppressed significantly by curcumin treatment in experimental colitis [25]. Although inhibition of downstream molecules such as cytokines, growth factors, interleukins and nitric oxide regulated by NF- $\kappa \mathrm{B}$ has been extensively studied, identification of upstream signaling molecules remains poorly understood.

Studies have demonstrated that curcumin can modulate both the proliferation and the activation of $\mathrm{T}$ cells. Curcumin inhibits the proliferation induced by concana- valin $\mathrm{A}$, phytohemagglutinin (PHA) and phorbol-12-myristate-13-acetate (PMA) of lymphocytes derived from fresh human spleen [35]. In addition, curcumin suppresses IL-2 synthesis and IL-2-induced proliferation of lymphocytes, suggesting that it exhibits immunosuppressive properties. In another study by the same authors, curcumin inhibits the proliferation induced by PMA and anti-CD28 antibody as well as the proliferation induced by PHA of T lymphocytes isolated from healthy donors [36]. In comparison, cyclosporine A suppresses PHA-induced T-cell proliferation but not that induced by PMA and anti-CD28 antibody. Thus, curcumin can overcome the resistance of PMA and CD28 pathway to cyclosporine A, highlighting its immunomodulating properties.

In addition to modulating $\mathrm{T}$-cell activity, curcumin influences the proliferation of B cells and B-lymphocytemediated immune function. Of note, curcumin blocks Epstein-Barr virus-induced immortalization of human B cells [37]. Apart from affecting normal cells, curcumin can induce apoptosis to reduce the proliferation of immature B-cell lymphoma (BKS-2) cells by downregulating egr-1, c-myc, bcl-XL, the tumor suppressor gene $p 53$, and by partial inhibition of NF- $\mathrm{\kappa B}$ activity [33].

Furthermore, studies have shown that curcumin can modulate the activation of macrophages, dependent on its ability to downregulate Thl and nitric oxide production [38]. Curcumin enhances the phagocytosis of peritoneal macrophages and differentially regulates the proliferation of splenocytes [39]. In addition, Joe and Lokesh [40] demonstrated that a daily diet of curcumin $(30 \mathrm{mg} /$ $\mathrm{kg}$ body weight/day) for 2 weeks in rats attenuates the ability of macrophages to generate reactive oxygen species and decreases the secretion of the lysosomal enzymes collagenase, elastase and hyaluronidase [41].

Neutrophils are important proinflammatory effector cells capable of associating with lymphocytes to foster epithelial dysfunction and injury associated with IBD. Impaired neutrophil recruitment has been implicated in the development of $C D$ wherein neutrophil accumulation and associated bacterial clearance are impaired and favor the formation of granulomatous inflammation [42]. In a recent study, curcumin modulates neutrophil motility by inhibiting the expression and production of chemoattractant molecules, macrophage inflammatory protein 2 , keratinocyte chemoattractant, macrophage inflammatory protein $1 \alpha$ and IL- $1 \beta$ by peritoneal macrophages and colonic epithelial cells. Moreover, in addition to altering the chemoattractant gradient formation, curcumin directly affects neutrophil chemotaxis [23]. 


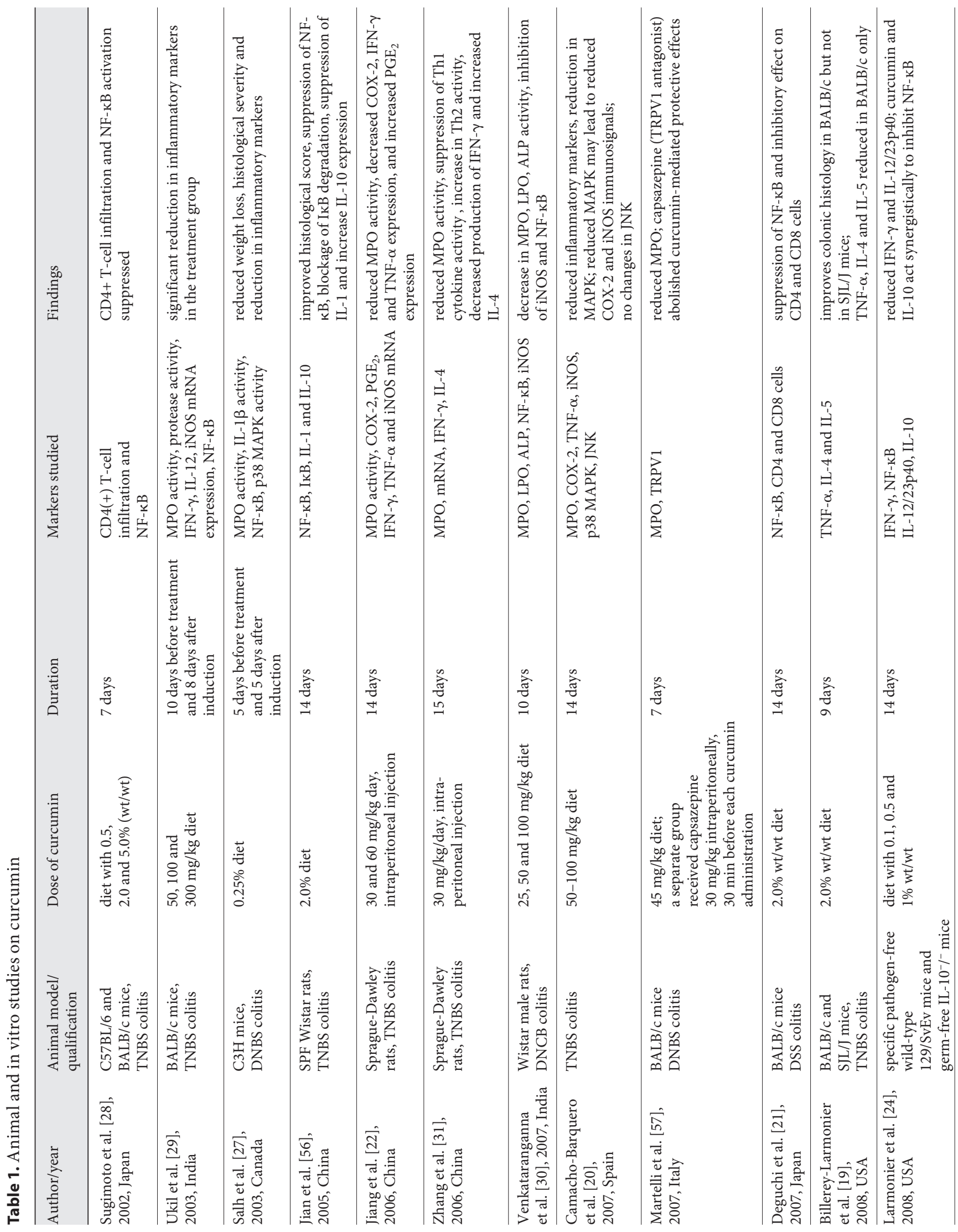




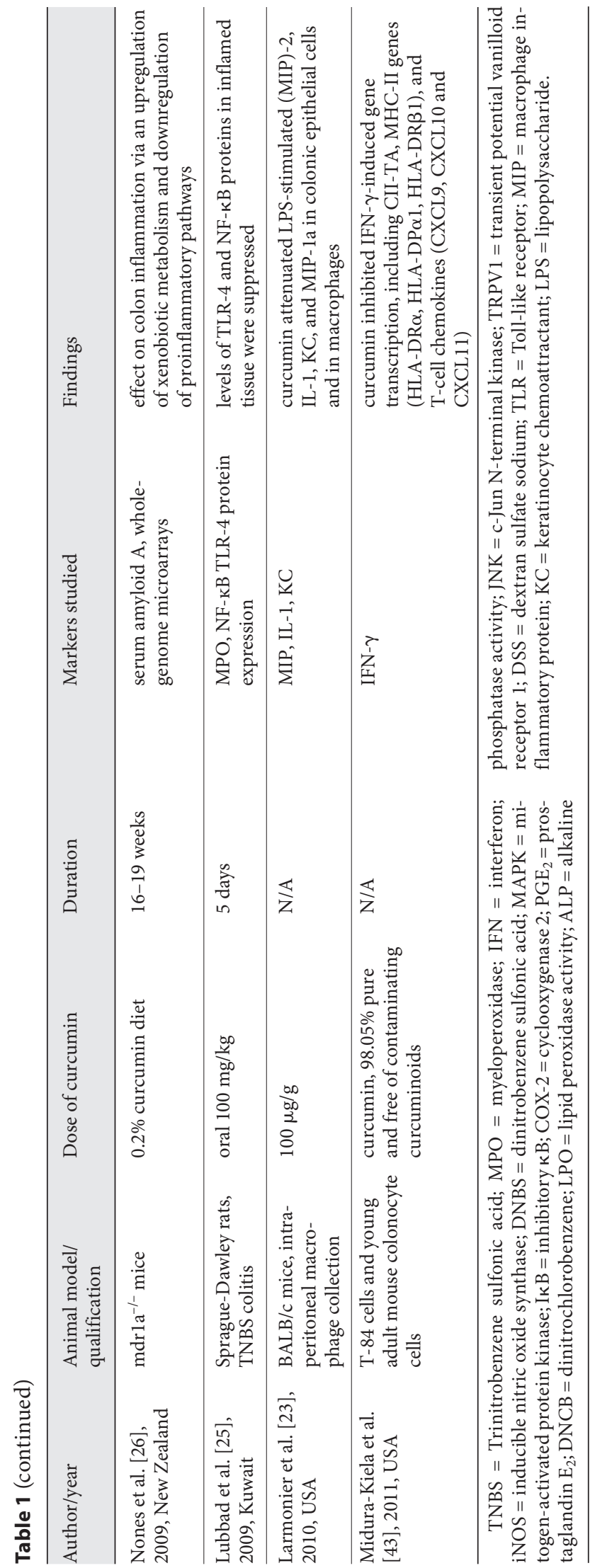

Table 2. Effects on cytokines by curcumin

\begin{tabular}{ll}
\hline Increased activity & Decreased activity \\
\hline PGE $_{2}[22]$ & NF-kB $[21,22,24,30,56]$ \\
IL-4 [31] & IFN- $\gamma[22,24,31,43]$ \\
IL-10 [56] & MAPK [20, 27] \\
& IL-1 [56], IL-4 [19], IL-5 [19] IL-12 [24, 29] \\
& TNF- $\alpha[19,20,22]$ \\
& MPO [20, 30, 31, 57] \\
& LPO [30] \\
& iNOS [20, 30] \\
& COX-2 [20, 22] \\
& TLR-4 [25]
\end{tabular}

$\mathrm{PGE}_{2}=$ Prostaglandin $\mathrm{E}_{2} ; \mathrm{IFN}-\gamma=$ interferon $-\gamma ; \mathrm{MAPK}=\mathrm{mi}-$ togen-activated protein kinase; $\mathrm{MPO}=$ myeloperoxidase; $\mathrm{LPO}=$ lipid peroxidase activity; iNOS = inducible nitric oxide synthase; COX-2 = cyclooxygenase 2 ; TLR- $4=$ Toll-like receptor 4 .

The intestinal epithelium, an essential component of the gut innate defense mechanisms, is profoundly affected by interferon- $\gamma$, which can disrupt the epithelial barrier function, prevent epithelial cell migration and wound healing, as well as prime epithelial cells to express major histocompatibility complex class II molecules and to serve as nonprofessional antigen-presenting cells [43]. Recently, curcumin has also been shown to act as an interferon- $\boldsymbol{\gamma}$-signaling inhibitor in colonocytes [43].

The effects of curcumin on various cytokines are summarized in table 2 .

\section{Human Studies with Curcumin}

Curcumin has been used for centuries in Asia for many inflammatory disorders, infections and digestive diseases [44]. More recently, its use as a therapeutic agent has been suggested for atherosclerosis, hyperlipidemia, thromboembolism, myocardial infarction, rheumatoid arthritis, HIV and cancers [45]. A few single-arm phase I studies have shown beneficial effects of curcumin in various malignancies. Curcumin is well tolerated without any serious toxicity and side effects. Several clinical studies have confirmed the safety of curcumin in humans with no treatment-related toxicity up to $8,000 \mathrm{mg} /$ day for 3 months [45, 46]. Minor gastrointestinal adverse events, including nausea and diarrhea, have been reported $[47,48]$.

The therapeutic effect of curcumin against a variety of chronic pathological conditions is likely driven by its an- 
Table 3. Clinical studies on curcumin

\begin{tabular}{|c|c|c|c|c|c|c|}
\hline $\begin{array}{l}\text { Author/ } \\
\text { year }\end{array}$ & $\begin{array}{l}\text { Type of study/ } \\
\text { patients }\end{array}$ & Disease & Dose of curcumin & Duration & Markers studied & Findings \\
\hline $\begin{array}{l}\text { Holt et al. } \\
\text { [52], 2005 }\end{array}$ & cohort/10 & $\begin{array}{l}\mathrm{UC} \\
\text { (proctitis-proctosigmoiditis) } \\
\text { and } \mathrm{CD}\end{array}$ & $\begin{array}{l}550 \mathrm{mg} \text { BID followed } \\
\text { by } 550 \mathrm{mg} \text { TID for UC } \\
\text { and } 360 \mathrm{mg} \text { TID } \\
\text { followed } \\
\text { by } 360 \mathrm{mg} \text { QID for CD }\end{array}$ & $\begin{array}{l}2 \text { months for } \mathrm{UC} \\
3 \text { months for } \mathrm{CD}\end{array}$ & $\begin{array}{l}\text { global scale (UC) } \\
\text { CDAI } \\
\text { ESR (CD) }\end{array}$ & $\begin{array}{l}\text { improved global scale } \\
(\mathrm{p}<0.02 \text {; UC) } \\
55 \text {-point decrease in } \\
\text { CDAI and } 10-\mathrm{mm} / \mathrm{h} \\
\text { drop in ESR }(\mathrm{CD})\end{array}$ \\
\hline
\end{tabular}

$\mathrm{BID}=$ Two times a day; TID = three times a day; QID = four times a day CDAI = Crohn's Disease Activity Index; ESR = erythrocyte sedimentation rate; $\mathrm{PO}$ = postoperatively; $\mathrm{CAI}=$ clinical activity index; $\mathrm{EI}=$ endoscopic index .

tioxidant and anti-inflammatory effect. The anti-inflammatory effect of curcumin is most likely mediated through its ability to inhibit enzymes that mediate inflammatory processes, such as cyclooxygenase 2 and lipoxygenase [49], as well as inducible nitric oxide synthase [50]. Therefore, the potent anti-inflammatory property of curcumin is anticipated to exert chemopreventive effects on carcinogenesis given a complex inter-relationship between inflammation and tumorigenesis [51]. The oral application of curcumin for a variety of inflammatory diseases has been reported in several human studies. Because curcumin plays a key role in the inhibition of proinflammatory cytokines, it could be used as a novel therapeutic agent in several inflammatory diseases, such as IBD. However, to date, there have only been two human studies with curcumin and IBD that have achieved encouraging results (table 3 ).

In a pilot study, Holt et al. [52] reported its use in ulcerative proctosigmoiditis and CD. Five patients with ulcerative proctitis were treated with $550 \mathrm{mg}$ curcumin twice daily for 1 month followed by $550 \mathrm{mg}$ three times daily for another month. All 5 patients improved as judged by global score $(\mathrm{p}<0.02)$, with reductions in concomitant medications in 4 patients. The 5 patients with $\mathrm{CD}$ were treated with $360 \mathrm{mg}$ curcumin three times daily for 1 month followed by $360 \mathrm{mg}$ four times daily for another 2 months. Four out of 5 patients with CD improved, as evidenced by lowered Crohn's Disease Activity Index, with a mean reduction of 55 points and a sedimentation rate with a mean of $10 \mathrm{~mm} / \mathrm{h}$ reduction.

Subsequently, Hanai et al. [53] evaluated the use of curcumin in 89 patients with quiescent UC in a randomized, double-blind, multicenter trial. Forty-five patients received $1 \mathrm{~g}$ curcumin twice a day along with sulfasala- zine or mesalamine, and 44 patients received placebo plus sulfasalazine or mesalamine for 6 months. Of 43 patients ( 2 patients violated the protocol) who received curcumin, 2 relapsed during 6 months of therapy (4.65\%), whereas 8 of 39 patients $(20.51 \%)$ in the placebo group relapsed ( $\mathrm{p}=$ 0.040 ). Recurrence rates evaluated on the basis of intention to treat showed a significant difference between curcumin and placebo $(p=0.049)$. Furthermore, curcumin improved both the clinical activity index $(\mathrm{p}=0.038)$ and the endoscopic index ( $\mathrm{p}=0.0001)$, measures used to evaluate the morbidity associated with UC. Based on these two studies, curcumin seems to be a promising and safe medication for maintaining remission in patients with quiescent $\mathrm{UC}$ as well as for improving symptoms in proctitis and CD. Further studies on curcumin and IBD are needed to strengthen these findings. The agent has the potential to be used as a steroid-sparing induction agent in mild to moderate colitis considering its effect on multiple inflammatory pathways. Alternatively, this agent can also be used as adjunct therapy in maintenance of remission in patients who encounter loss of response to an agent directed against a single cytokine such as antiTNF agents.

\section{Limitations and Conclusion}

Despite the promising biological effects of curcumin, its poor oral bioavailability in both rodents and humans [54] has restricted its use in the management of human ailments. It is well known that many drugs have bioavailability problems due to their low water solubility, slow dissolution rate and instability in the gastrointestinal tract. Poor oral absorption due to its extremely low aque- 
ous solubility or extensive presystemic metabolism may be responsible for the unfavorable pharmacokinetics of this molecule. In rodents, curcumin undergoes avid metabolism by conjugation and reduction, and its absorption after oral dosing is characterized by poor systemic bioavailability [55]. One study has used the cyclodextrin complex of curcumin to increase its solubility and dissolution that may overcome the pharmacokinetic problems [14]. New formulations are needed to increase the bioavailability of curcumin.

Furthermore, well-designed randomized clinical trials with large cohorts will be needed to validate the results of previous studies and evaluate the full clinical potential of curcumin in the treatment of human disease. Currently, several clinical trials are being conducted on the efficacy of curcumin in a variety of diseases, with two involving IBD. One is a recently completed phase I clinical trial seeking to determine the tolerability of curcumin in pediatric patients with IBD. However, results of this study are pending. Another trial aims to study the efficacy and tolerability of Coltect, which contains curcumin, green tea and selenium, as an add-on in patients with active UC. For details, please refer to www.clinicaltrials.gov.

The key clinical question will be to define its role in the current therapeutic armentarium of IBD. Studies to explore its role as a steroid-sparing induction agent in mild to moderate colitis or as an adjunct or rescue therapy to maintain remission in patients who are losing re- sponse to agents such as immunomodulators or anti-TNF agents will be needed in near future.

In conclusion, the increase in patients' use of CAM has prompted substantial interest in CAM among gastroenterologists and other physicians who care for patients with IBD. Their side effect profiles, including serious infections and risk of lymphomas, have led to interest in natural anti-inflammatory compounds such as curcu$\mathrm{min}$, derived from turmeric. The current medical therapy for IBD patients includes agents such as mesalamine, steroids and anti-TNF agents. These agents are associated with potential side effects including increased risk of serious infections and malignancies. The efficacy of these agents in inducing response and remission is also somewhat limited. One of the reasons to lose response to therapy such as anti-TNF therapy is a shift in the inflammatory pathway in which TNF may not be a major cy tokine. That has led to some recent research exploring other cytokine pathways (e.g., IL-6) to control disease processes. Curcumin has been found to inhibit many of these cytokine pathways including IL- 6 , and thus, is an important natural compound that carries minimal toxicity with a favorable safety profile. Its anti-inflammatory and antioxidant effect have been shown in numerous animal models. However, lack of controlled human studies may limit its use in clinical practice. Further prospective trials of curcumin in the near future may help us determine its potential beneficial role in the management algorithm of the IBD patients.

\section{References}

$\checkmark 1$ Hanauer SB: Inflammatory bowel disease. N Engl J Med 1996;334:841-848.

$\checkmark 2$ Podolsky DK: Inflammatory bowel disease. N Engl J Med 2002;347:417-429.

3 Rutgeerts P, Van Assche G, Vermeire S: Optimizing anti-TNF treatment in inflammatory bowel disease. Gastroenterology 2004; 126:1593-1610.

4 Galloway JB, et al: Anti-TNF therapy is associated with an increased risk of serious infections in patients with rheumatoid arthritis especially in the first 6 months of treatment: updated results from the British Society for Rheumatology Biologics Register with special emphasis on risks in the elderly. Rheumatology (Oxford) 2011;50:124-131.

5 Afif W, Loftus EV Jr: Safety profile of IBD therapeutics: infectious risks. Med Clin North Am 2010;94:115-133.
Beaugerie L, et al: Lymphoproliferative disorders in patients receiving thiopurines for inflammatory bowel disease: a prospective observational cohort study. Lancet 2009;374: 1617-1625.

7 Barnes PM, Bloom B, Nahin R: CDC National Health Statistics Report No. 12. Complementary and alternative medicine use among adults and children: United States 2007. Hyattsville, National Center for Health Statistics, 2008.

8 Hilsden RJ, et al: Use of complementary and alternative medicine by patients with inflammatory bowel disease. Inflamm Bowel Dis 2011;17:655-662.

9 Ruby AJ, et al: Anti-tumour and antioxidant activity of natural curcuminoids. Cancer Lett 1995;94:79-83.

10 Ramsewak RS, DeWitt DL, Nair MG: Cytotoxicity, antioxidant and anti-inflammatory activities of curcumins I-III from Curcuma longa. Phytomedicine 2000;7:303-308.
11 Aggarwal BB, Harikumar KB: Potential therapeutic effects of curcumin, the anti-inflammatory agent, against neurodegenerative, cardiovascular, pulmonary, metabolic, autoimmune and neoplastic diseases. Int J Biochem Cell Biol 2009;41:40-59.

12 Rao DS, et al: Effect of curcumin on serum and liver cholesterol levels in the rat. J Nutr 1970;100:1307-1315.

13 Negi PS, et al: Antibacterial activity of turmeric oil: a byproduct from curcumin manufacture. J Agric Food Chem 1999;47:42974300.

14 Yadav VR, et al: Effect of cyclodextrin complexation of curcumin on its solubility and antiangiogenic and anti-inflammatory activity in rat colitis model. AAPS Pharm Sci Tech 2009;10:752-762

15 Subramanian L, Selvam R: Prevention of CCI4-induced hepatotoxicity by aqueous extract of turmeric. Nutr Res 1999;19:429-441. 
16 Wilczynski NL, Haynes RB: Developing optimal search strategies for detecting clinically sound prognostic studies in MEDLINE: an analytic survey. BMC Med 2004;2:23.

17 Haynes RB, et al: Developing optimal search strategies for detecting clinically sound studies in MEDLINE. J Am Med Inform Assoc 1994;1:447-458.

18 Haynes RB, Kastner M, Wilczynski NL: Developing optimal search strategies for detecting clinically sound and relevant causation studies in EMBASE. BMC Med Inform Decis Making 2005;5:8.

19 Billerey-Larmonier C, et al: Protective effects of dietary curcumin in mouse model of chemically induced colitis are strain dependent. Inflamm Bowel Dis 2008;14:780-793.

20 Camacho-Barquero L, et al: Curcumin, a Curcuma longa constituent, acts on MAPK p38 pathway modulating COX-2 and iNOS expression in chronic experimental colitis. Int Immunopharmacol 2007;7:333-342.

21 Deguchi Y, et al: Curcumin prevents the development of dextran sulfate sodium (DSS)induced experimental colitis. Dig Dis Sci 2007;52:2993-2998.

22 Jiang H, et al: Curcumin-attenuated trinitrobenzene sulphonic acid induces chronic colitis by inhibiting expression of cyclooxygenase-2. World J Gastroenterol 2006;12:38483853.

23 Larmonier CB, et al: Modulation of neutrophil motility by curcumin: implications for inflammatory bowel disease. Inflamm Bowel Dis 2011;17:503-515.

24 Larmonier CB, et al: Limited effects of dietary curcumin on Th-1 driven colitis in IL10 deficient mice suggest an IL-10-dependent mechanism of protection. Am J Physiol Gastrointest Liver Physiol 2008;295:G1079_ G1091.

25 Lubbad A, Oriowo MA, Khan I: Curcumin attenuates inflammation through inhibition of TLR-4 receptor in experimental colitis. Mol Cell Biochem 2009;322:127-135.

26 Nones K, et al: The effects of dietary curcumin and rutin on colonic inflammation and gene expression in multidrug resistance gene-deficient (mdrla-/-) mice, a model of inflammatory bowel diseases. Br J Nutr 2009;101:169-181.

27 Salh B, et al: Curcumin attenuates DNB-induced murine colitis. Am J Physiol Gastrointest Liver Physiol 2003;285:G235-G243.

28 Sugimoto K, et al: Curcumin prevents and ameliorates trinitrobenzene sulfonic acidinduced colitis in mice. Gastroenterology 2002;123:1912-1922.
9 Ukil A, et al: Curcumin, the major component of food flavour turmeric, reduces mucosal injury in trinitrobenzene sulphonic acid-induced colitis. Br J Pharmacol 2003; 139:209-218.

30 Venkataranganna MV, et al: NCB-02 (standardized Curcumin preparation) protects dinitrochlorobenzene-induced colitis through down-regulation of NFkappa-B and iNOS. World J Gastroenterol 2007;13:11031107.

31 Zhang M, et al: Curcumin regulated shift from Th1 to Th2 in trinitrobenzene sulphonic acid-induced chronic colitis. Acta Pharmacol Sin 2006;27:1071-1077.

32 Cuendet M, Pezzuto JM: The role of cyclooxygenase and lipoxygenase in cancer chemoprevention. Drug Metabol Drug Interact 2000;17:109-157.

33 Han SS, et al: Curcumin causes the growth arrest and apoptosis of $\mathrm{B}$ cell lymphoma by downregulation of egr-1, c-myc, bcl-XL, NFkappa B, and p53. Clin Immunol 1999;93: 152-161.

34 Boone DL, Ma A: Connecting the dots from Toll-like receptors to innate immune cells and inflammatory bowel disease. J Clin Invest 2003;111:1284-1286.

35 Ranjan D, et al: Curcumin inhibits mitogen stimulated lymphocyte proliferation, NFkappaB activation, and IL-2 signaling. J Surg Res 2004;121:171-177.

36 Ranjan D, et al: Curcumin blocks cyclosporine A-resistant CD28 costimulatory pathway of human T-cell proliferation. J Surg Res 1998;77:174-178.

37 Jagetia GC, Aggarwal BB: 'Spicing up' of the immune system by curcumin. J Clin Immunol 2007;27:19-35.

38 Bhaumik S, Jyothi MD, Khar A: Differential modulation of nitric oxide production by curcumin in host macrophages and NK cells. FEBS Lett 2000;483:78-82.

39 Xinjian L, Xiaocheng L: Effect of curcumin on immune function of mice. J Huazhong Univ Sci Technol Med Sci 2005;25:137-140.

40 Joe B, Lokesh BR: Role of capsaicin, curcumin and dietary $n-3$ fatty acids in lowering the generation of reactive oxygen species in rat peritoneal macrophages. Biochim Biophys Acta Mol Cell Res 1994;1224:255-263.

41 Joe B, Lokesh B: Dietary n-3 fatty acids, curcumin and capsaicin lower the release of lysosomal enzymes and eicosanoids in rat peritoneal macrophages. Mol Cell Biochem 2000;203:153-161.

42 Marks DJ, Segal AW: Innate immunity in inflammatory bowel disease: a disease hypothesis. J Pathol 2008;214:260-266.

43 Midura-Kiela MT, et al: Curcumin inhibits interferon-gamma signaling in colonic epithelial cells. Am J Physiol Gastrointest Liver Physiol 2012;302:G85-G96.
44 Hanai $\mathrm{H}$, Sugimoto K: Curcumin has bright prospects for the treatment of inflammatory bowel disease. Curr Pharm Des 2009;15: 2087-2094.

-45 Hsu CH, Cheng AL: Clinical studies with curcumin. Adv Exp Med Biol 2007;595:471480

46 Cheng AL, et al: Phase I clinical trial of curcumin, a chemopreventive agent, in patients with high-risk or pre-malignant lesions. Anticancer Res 2001;21:2895-2900.

47 Sharma RA, et al: Pharmacodynamic and pharmacokinetic study of oral Curcuma extract in patients with colorectal cancer. Clin Cancer Res 2001;7:1894-1900.

48 Sharma RA, et al: Phase I clinical trial of oral curcumin: biomarkers of systemic activity and compliance. Clin Cancer Res 2004;10: 6847-6854.

49 Rao CV: Regulation of COX and LOX by curcumin. Adv Exp Med Biol 2007;595:13-26.

50 Prakobwong S, et al: Curcumin decreases cholangiocarcinogenesis in hamsters by suppressing inflammation-mediated molecular events related to multistep carcinogenesis. Int J Cancer 2011;129:88-100.

51 Menon VP, Sudheer AR: Antioxidant and anti-inflammatory properties of curcumin. Adv Exp Med Biol 2007;595:105-125.

52 Holt PR, Katz S, Kirshoff R: Curcumin therapy in inflammatory bowel disease: a pilot study. Dig Dis Sci 2005;50:2191-2193.

53 Hanai $\mathrm{H}$, et al: Curcumin maintenance therapy for ulcerative colitis: randomized, multicenter, double-blind, placebo-controlled trial. Clin Gastroenterol Hepatol 2006;4:15021506.

54 Pan MH, Huang TM, Lin JK: Biotransformation of curcumin through reduction and glucuronidation in mice. Drug Metab Dispos 1999;27:486-494.

55 Holder GM, Plummer JL, Ryan AJ: The metabolism and excretion of curcumin (1,7-bis(4-hydroxy-3-methoxyphenyl)-1,6-heptadiene-3,5-dione) in the rat. Xenobiotica 1978;8:761-768.

56 Jian YT, et al: Preventive and therapeutic effects of NF-kappaB inhibitor curcumin in rats colitis induced by trinitrobenzene sulfonic acid. World J Gastroenterol 2005; 11 : 1747-1752.

57 Martelli L, et al: A potential role for the vanilloid receptor TRPV1 in the therapeutic effect of curcumin in dinitrobenzene sulphonic acid-induced colitis in mice. Neurogastroenterol Motil 2007;19:668-674. 